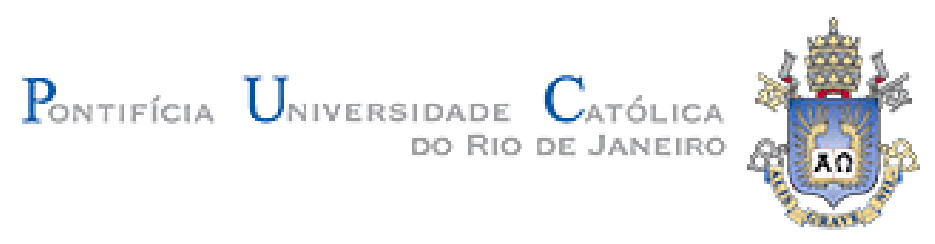

Rafael Salomão Ach

\title{
Influência da taxa de deformação nas curvas tensão x deformação de um aço 1020 pré-encruado
}

Dissertação de Mestrado

Dissertação apresentada como requisito parcial para obtenção do grau de Mestre pelo Programa de Pósgraduação em Engenharia Mecânica do Departamento de Engenharia Mecânica da PUC-Rio.

Orientador: Prof. Jaime Tupiassú Pinho de Castro 


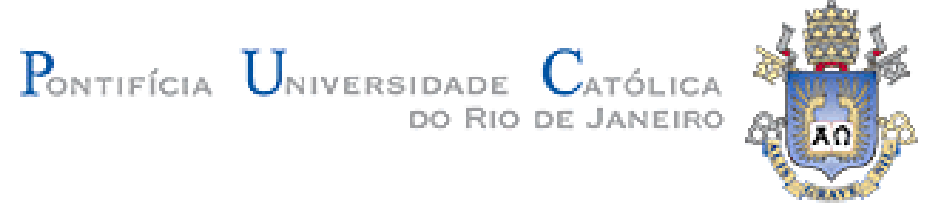

Rafael Salomão Ach

\begin{abstract}
Influência da taxa de deformação nas curvas tensão x deformação de um aço 1020 pré-encruado
\end{abstract}

Dissertação apresentada como requisito parcial para a obtenção do grau de Mestre pelo Programa de PósGraduação em Engenharia Mecânica da PUC-Rio. Aprovada pela Comissão Examinadora abaixo assinada.

Prof. Jaime Tupiassú Pinho de Castro

Orientador

Departamento de Engenharia de Mecânica - PUC-Rio

Prof. Marco Antônio Meggiolaro Departamento de Engenharia de Mecânica - PUC-Rio

Prof. José Luiz de França Freire Departamento de Engenharia de Mecânica - PUC-Rio

Prof. Paulo Pedro Kenedi CEFET/RJ

Prof. José Eugenio Leal Coordenador Setorial do Centro Científico - PUC-Rio 
Todos os direitos reservados. É proibida a reprodução total ou parcial do trabalho sem autorização da universidade, do autor e do orientador.

\section{Rafael Salomão Ach}

É Primeiro Tenente (EN) da Marinha do Brasil desde 2011 atuando como coordenador de reparo e projetos de submarinos. Graduado em Engenheiro Mecânico pelo CEFET-RJ, em 2004 e PósGraduado em Engenharia de Dutos pela PUC RJ em 2006. Especializado em Análise de Falha pela ABM e certificado pela Instituição INDG em Green Belt, ambos em 2007.

Ficha Catalográfica

Ach, Rafael Salomão

Influência da taxa de deformação nas curvas tensão $x$ deformação de um aço 1020 pré-encruado. / Rafael Salomão Ach; orientador: Jaime Tupiassú Pinho de Castro - Rio de Janeiro: PUC, Departamento de Engenharia Mecânica, 2012.

v.,105 f,; il. ; $29,7 \mathrm{~cm}$

1. Dissertação (Mestrado) - Pontifícia Universidade Católica do Rio de Janeiro, Departamento de Engenharia Mecânica.

Inclui referências bibliográficas.

1. Engenharia mecânica - Dissertação. 2. Influência da Taxa de Deformação. 3. Ensaio de Tração. 4. Comportamento Mecânico Dinâmico. 5 Ajuste Ótimo de Dados.I. Castro, Jaime Tupiassú Pinho de. II. Pontifícia Universidade Católica do Rio de Janeiro. Departamento de Engenharia Mecânica. III Título.

CDD: 621 
Para minha família. 


\section{Agradecimentos}

À Deus, que me concede bênçãos, dentre elas, a de poder estudar.

A Alice Salomão de Freitas, pelo incentivo na minha formação acadêmica (in memorian)

Aos meus pais e irmãos, que sempre me incentivaram aos estudos e são o alicerce da minha formação pessoal.

A Liz Villacorta, por ser especial e importante para mim.

Ao Professor Jaime Tupiassú Pinho de Castro - Ph.D., pela ajuda, rigor acadêmico, orientação, amizade e confiança.

Ao Professor Marco Antonio Meggiolaro - Ph.D., pela compreensão no reingresso ao curso de Mestrado.

Ao amigo que chamo carinhosamente de Jaiminho, engenheiro civil e mestrando na PUC -RJ, que muito me ajudou na parte experimental durante meses de testes no laboratório de fadiga - Departamento de Engenharia Mecânica da PUC RJ. 


\section{Resumo}

Ach, Rafael Salomão; Castro, Jaime Tupiassú Pinho de. Influência da taxa de deformação nas curvas tensão $x$ deformação de um aço 1020 préencruado. Rio de Janeiro, 2012. 105p. Dissertação de Mestrado Departamento de Engenharia Mecânica, Pontifícia Universidade Católica do Rio de Janeiro.

Curvas tensão $\times$ deformação $\sigma \times \varepsilon$ de um aço 1020 trabalhado a frio foram obtidas sob controle da taxa de deformação imposta no corpo de prova (e não da velocidade do pistão ou do travessão da máquina de testes), no intervalo $10^{-5} \leq \dot{\boldsymbol{\varepsilon}}$ $\leq 3 \mathrm{~s}^{-1}$. Este amplo conjunto de dados, tratado por um versátil programa desenvolvido para adquiri-los, filtrá-los e apresentá-los em tempo real, foi usado para verificar a adequação de diversos modelos propostos para descrever o efeito desta taxa, aplicando um algoritmo de otimização de Levenberg-Marquardt para ajustar os vários modelos estudados ao conjunto dos dados medidos. Este procedimento relativamente simples elimina os erros sistemáticos inerentes à prática tradicional de medir o efeito da taxa de deformação nas curvas $\sigma \times \varepsilon$ controlando a velocidade da carga, que podem ser particularmente relevantes nos corpos de prova de aço, cuja rigidez muitas vezes é pelo menos uma ordem de grandeza maior do que a da máquina de testes.

\section{Palavras-chave}

Influência da taxa de deformação; comportamento mecânico dinâmico; ajuste ótimo de dados. 


\section{Abstract}

Ach, Rafael Salomão; Castro, Jaime Tupiassú Pinho de (Advisor). Strain rate influence on the stress $x$ strain behavior of a cold worked 1020 steel. Rio de Janeiro, 2012. 105p. MSc. Dissertation - Departamento de Engenharia Mecânica, Pontifícia Universidade Católica do Rio de Janeiro.

Stress $\times$ strain $\sigma \times \varepsilon$ curves of a cold worked 1020 steel were measured controlling the strain-rate imposed on the test specimens (and not the testing machine piston or load bean speed), in the range $10^{-5} \leq \dot{\boldsymbol{\varepsilon}} \leq 3 \mathrm{~s}^{-1}$. This ample data set, properly treated by a versatile software developed to acquire, filter and present the data in real time, was used to verify the adequacy of several models proposed to describe the strain-rate influence, using an Levenberg-Marquardt optimization algorithm to fit the models to the ensemble of the measured data. This relatively simple procedure avoids the intrinsic systematic errors associated to the traditional practice of measuring the strain-rate effect on the $\sigma \times \varepsilon$ curves controlling the load speed, which may be particularly relevant when testing steel specimens, as their stiffness is many times at least an order of magnitude greater than those of the testing machines.

\section{Keywords}

Strain rate effect; dynamic mechanical behavior; optimal data fitting. 


\section{Sumário}

1. Introdução 17

1.1. Motivação 20

1.2. Revisão da Literatura 21

1.2.1. Influência da Máquina de Teste nas Propriedades

$\begin{array}{ll}\text { Mecânicas } & 29\end{array}$

2. Procedimento para Teste e Procedimentos para Ajuste 30

2.1. Procedimento para Teste 30

2.1.1. Determinação do Número de Corpos de Prova 30

2.1.2. Caracterização do Material e Rastreabilidade 31

2.1.3. Descrição da Máquina de Ensaio e Corpo de Prova 31

2.1.4. Parametrização dos Ensaios de tração 33

2.1.5. Razões para Ensaio de Tração Real e suas

Propriedades Mecânicas 35

2.2. Metodologia de Ajuste de Dados Experimentais 37

2.3. Metodologia para Determinação de $S_{E} \quad 40$

3. Analise dos Modelos Matemáticos 42

3.1. Introdução 42

3.1.1. Modelos de Johnson-Cook 42

3.1.2. Modelos de Meyers 43

3.1.3. Modelos de Zerilli-Armstrong 44

3.1.4. Modelos de Cowper-Symonds 44

4. Resultados Experimentais 46

4.1. Introdução 46 
4.2. Resultados Gerais dos Ensaios de Tração por Controle de Deformação

4.2.1.Propriedades Mecânicas em Função do Efeito

da Taxa de Deformação

4.2.2. Valores das Propriedades Mecânicas $S_{E}$ e $S_{R}$

para os Aços 1020 Pré encruado e Recozido

4.3 Considerações dos Modelos Matemáticos quanto ao

Ajuste dos Resultados Experimentais 51

4.3.1. Resultados do Modelo de Johnson Cook 52

4.3.2. Resultados do Modelo de Meyers 56

4.3.3. Resultados do Modelo de Zerilli-Armstrong 60

4.3.4. Resultados do Modelo Cowper-Symonds 64

4.3.5. Comparação dos Resultados dos Modelos Matemáticos 68

5. Conclusões e Sugestões para Trabalhos Futuros 72

5.1. Conclusão das Propriedades Mecânicas 72

5.2. Conclusões do Efeito da Taxa de Deformação nos valores $\begin{array}{ll}\text { de } S_{E} \text { e } S_{R} & 74\end{array}$

5.3. Conclusões dos Modelos Matemáticos 76

5.4. Considerações Finais Sobre o Efeito de Taxa de Deformação em Projeto Mecânico de Dutos 84

5.5. Sugestões para Trabalhos Futuros 89

$\begin{array}{ll}\text { 6. Bibliografia } & 90\end{array}$

$\begin{array}{ll}\text { Apêndice A } & 93\end{array}$ 


\section{Lista de Figuras}

Figura 1 - Esquema básico do sistema de controle de uma máquina servo-hidráulica

Figura 2 - Deslocamento do pistão y e deformação (de engenharia)

$\varepsilon$ atuante no CP durante um ensaio feito sob taxa de deformação

Constante

Figura 3 - Deslocamento do pistão e deformação atuante num CP

idêntico ao da figura 2, durante um ensaio feito sob velocidade do pistão fixa

Figura 4 - Relação de tipos de máquinas de tração com a faixa de taxa de deformação

Figura 5 - Gráfico tensão x deformação nas taxas de deformação suscetíveis a colisões veiculares segundo Chen (2004)

Figura 6 - Comportamento do aço HSLA em ensaio de tração submetidos a diversas temperaturas de ensaio. Thompson (2006)

Figura 7 - Dificuldade em ajustar a resposta gráfica para altas taxas no estudo da Arcelor Mittal

Figura 8 - Comportamento do aço EDDS, DP 590 CR

e DP 780 EG da ArcelorMittal

Figura 9 - Adequação do modelo de Johnson e Cook para

Alumínio 5083

Figura 10 - Adequação do modelo de Zerilli e Armstrong para Alumínio 5083

Figura 11 - Comportamento dinâmico de diferentes ligas de aço de aplicação balística

Figura 12 - Componentes de incerteza independentes do material 1 
Figura 13 - Componentes de incerteza independentes do material $2 \quad 28$

Figura 14 - Conjunto garra, corpo de prova e clip gage 32

Figura 15 - Painel de Controle da Instron modelo 850133

Figura 16 - Corpo de prova segundo norma ABNT 6152

Figura 17 - Tela de acompanhamento dos ensaios de tração em tempo real 35

Figura 18 - Ajuste de dados para o aço 300M e API 5L-X60 39

Figura 19 - Determinação de $S_{E}$ para os ensaios

experimentais e modelos matemáticos

Figura 20 - As 13 curvas $\sigma \times \varepsilon$ de engenharia medidas

nas várias. $\dot{\varepsilon}$

Figura 21 - Detalhe da região plástica das curvas

$\sigma \times \varepsilon$ reais (até a estricção)

Figura 22 - Relação de $S_{R}$ dos aços 1020 pré encruado

e recozido com $\dot{\varepsilon}$

Figura 23 - Relação de JC modificado com dados experimentais 55

Figura 24 - Erro de $S_{E}$ para a equação de JC 53

Figura 25 - Erro de $S_{R}$ para a equação de JC 54

Figura 26 - Relação de Meyers modificado com dados experimentais 59

Figura 27 - Erro de $S_{E}$ para a equação de Meyers 57

Figura 28 - Erro de $S_{R}$ para a equação de Meyers 57

Figura 29 - Relação de ZA modificado com dados experimentais 63

Figura 30 - Erro de $S_{E}$ para a equação de ZA 61

Figura 31 - Erro de $S_{R}$ para a equação de ZA 61

Figura 32 - Relação de CS modificado com dados experimentais $\quad 67$

Figura 33 - Erro de $S_{E}$ para a equação de CS 65

Figura 34 - Erro de $S_{R}$ para a equação de CS 65

Figura 35 - Erro percentual de $\mathrm{S}_{\mathrm{E}}$ para os modelos matemáticos $\quad 69$ 
Figura 36 - Erro percentual de $S_{R}$ para os modelos matemáticos

Figura 37 - Patamar de encruamento de JC

Figura 38 - Patamar de encruamento de MEYERS

Figura 39 - Patamar de encruamento de ZA

82

Figura 40 - Patamar de encruamento de CS

82

Figura 41 - Valor de $S_{E}$ para aço API 5L X52 


\section{Lista de Tabelas}

Tabela 1 - Comparação entre aço SAE 1020 recozido versus pré-encruado na taxa de referência

Tabela 2 - Taxas de deformação real usadas nos ensaios de tração

Tabela 3 - Constantes ajustadas por LM

Tabela 4 - Resistências ao escoamento e resistência máxima em função da taxa $\dot{\varepsilon}$

Tabela 5. Resistências ao escoamento e resistência máxima validados em função $\dot{\varepsilon}$

Tabela 6 - Sensibilidade de $S_{E}$ e $S_{R}$ em função $\dot{\varepsilon}$

Tabela 7 - Propriedades Mecânicas em função das

taxas de deformação

Tabela 8 - Variação das Propriedades Mecânicas em função das taxas de deformação

Tabela 9 - Comparação entre o Aço SAE 1020 Pré Encruado

e Recozido

Tabela 10 - Resultado de ERRO e $\mathrm{R}^{2}$ para JC modificado

Tabela 11 - Comparação de $S_{E}$ e $S_{R}$ de JC modificado

com resultados experimentais

Tabela 12 - Resultado de ERRO e $\mathrm{R}^{2}$ para Meyers modificado

Tabela 13 - Comparação de $S_{E}$ e $S_{R}$ de Meyers modificad

com resultados experimentais

Tabela 14 - Resultado de ERRO e $\mathrm{R}^{2}$ para ZA modificado 
Tabela 15 - Comparação de $S_{E}$ e $S_{R}$ de ZA modificado $\begin{array}{ll}\text { com resultados experimentais } & 60\end{array}$

Tabela 16 - Resultado de ERRO e $\mathrm{R}^{2}$ para CS modificado 64

Tabela 17 - Comparação de $S_{E}$ e $S_{R}$ de CS modificado com resultados experimentais $\quad 64$

Tabela 18 - Reunião de $S_{E}$ e $S_{R}$ para os modelos matemáticos (MPa) 68

Tabela 19 - Diferença percentual entre de $S_{E}$ e $S_{R}$ dos modelos matemáticos e experimentais (\%)

Tabela 20 - Comparação das propriedades mecânicas em função das taxas de deformação

Tabela 21 - Comparação das propriedades mecânicas em função das taxas de deformação

Tabela 22 - Comparação da sensibilidade de $S_{E}$ e $S_{R}$ em função

$\dot{\varepsilon}$ dos aços 1020 recozido e pré-encruado

Tabela 23 - Desempenho em vários aspectos dos modelos Matemáticos

Tabela 24 - Avaliação da região conservativa dos modelos Matemáticos

Tabela 25 - Variação dos coeficientes de segurança de TRESCA e MISES 


\section{Lista de Símbolos}

$\dot{\mathcal{E}}_{e}$ - Taxa de deformação na região elástica $\left(\mathrm{s}^{-1}\right)$

$\dot{\varepsilon}$ - Taxa de deformação (\%/s)

$\dot{\varepsilon}_{0}$ - Taxa de deformação de referência $\left(\mathrm{s}^{-1}\right)$

$\dot{\mathcal{E}}_{m}$ - Taxa de deformação média $\left(\mathrm{s}^{-1}\right)$

$\dot{\mathcal{E}}_{p}$ - Taxa de deformação na região plástica $\left(\mathrm{s}^{-1}\right)$

$\Omega$ - Margem de erro

$\phi$ MISES - Coeficiente de segurança de MISES

$\phi$ TRESCA - Coeficiente de segurança de TRESCA

$A$ - Área da seção resistente $\left(\mathrm{m}^{2}\right)$

$A_{R}$ - Alongamento Real (\%)

$A_{R}$ - Ductilidade real

$\mathrm{B}, \mathrm{N}, \mathrm{C}$ - Constantes dos modelos matemáticos

D - Diâmetro (mm)

e - Espessura (mm)

$E$ - Módulo de elasticidade (GPa)

F - Fator de construção

k - Rigidez da máquina (N/m)

K- Coeficiente de resistência plástica (MPa)

$\mathrm{K}^{\prime}$ - Constante do material (MPa/s)

$L_{0}$ - Comprimento efetivo do CP (m)

m - Fator de sensibilidade da taxa de deformação

$\mathrm{N}$ - Número de corpo de prova

$P$ - Carga aplicada(N)

$P_{d}$ - Pressão de projeto (MPa)

$\mathrm{P}_{\mathrm{t}}$ - Pressão de teste (MPa)

$R^{2}$ - Coeficiente de determinação 
S - Desvio padrão

$S^{\prime}$ - Fator da classe do tubo

$\mathrm{S}_{\mathrm{E}}$ - Resistência ao escoamento da curva real (MPa)

$\mathrm{S}_{\mathrm{e}}$ - Resistência ao escoamento da curva convencional (MPa)

$\mathrm{S}_{E 0}$ - Resistência ao escoamento medida na taxa de referência (MPa)

$S_{R}$ - Resistência máxima da curva real (MPa)

$\mathrm{S}_{\mathrm{R}}$ - Resistência máxima (MPa)

$\mathrm{S}_{\mathrm{r}}$ - Resistência máxima da curva convencional (MPa)

$\mathrm{T}$ - Fator de temperatura

$t$ - Tempo (s)

$\mathrm{U}_{\mathrm{T}}$ - Tenacidade real $(\mathrm{Pa})$

$v$ - Velocidade do travessão $(\mathrm{m} / \mathrm{s})$

$X$ - Número filtrado de pontos obtidos pelo experimento

Z $\alpha / 2$ - Grau de confiabilidade

$\varepsilon$ - Deformação $(\mathrm{mm} / \mathrm{mm})$

$\varepsilon_{A}$ - Deformação real no ponto $A$

$\varepsilon_{B}-$ Deformação real no ponto $B$

$\varepsilon_{E}$ - Deformação associada a resitência ao escoamento $(\mathrm{m} / \mathrm{m})$

$\varepsilon_{p}$ - Deformação plástica $(\mathrm{m} / \mathrm{m})$

$\varepsilon_{R}$ - Deformação real $(\mathrm{m} / \mathrm{m})$

$\varepsilon_{\text {uts }}$ - Alongamento até a resistência máxima da curva tensão deformação

convencional (\%)

$\sigma$ - Tensão (MPa)

$\sigma_{1}$ - Tensão na direção $1(\mathrm{MPa})$

$\sigma_{2}-$ Tensão na direção 2(MPa)

$\sigma_{3}-$ Tensão na direção $3(\mathrm{MPa})$

$\sigma_{A}-$ Tensão real no ponto $A$

$\sigma_{B}-$ Tensão real no ponto $B$

$\sigma$ calculado - Tensão calculada pelos modelos matemáticos (MPa)

$\sigma$ eq MISES - Tensão equivalente de MISES (MPa)

$\sigma$ eq TRESCA - Tensão equivalente de TRESCA (MPa)

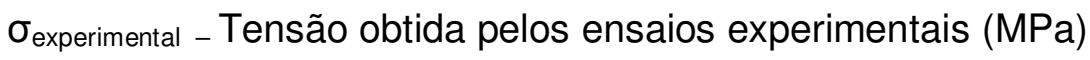

$\sigma_{R}$-Tensão real (MPa) 